\title{
Inhibition of Opioid Transmission at the $\mu$-Opioid Receptor Prevents Both Food Seeking and Binge-Like Eating
}

\author{
Chiara Giuliano*,', Trevor W Robbins', Pradeep J Nathan ${ }^{2,3}$, Edward T Bullmore ${ }^{1,2,3}$ and Barry J Everitt' \\ 'Behavioral and Clinical Neuroscience Institute and Department of Experimental Psychology, University of Cambridge, Cambridge, UK; \\ ${ }^{2}$ Department of Psychiatry, University of Cambridge, Addenbrooke's Hospital, Cambridge, UK; ${ }^{3}$ Clinical Unit Cambridge and Academic DPU, \\ GlaxoSmithKline R\&D, Clinical Unit Cambridge, Addenbrooke's Hospital, Cambridge, UK
}

\begin{abstract}
Endogenous opioids, and in particular $\mu$-opioid receptors, have been linked to hedonic and rewarding mechanisms engaged during palatable food intake. The aim of this study was to investigate the effects of GSK I52 I 498, a novel $\mu$-opioid receptor antagonist, on foodseeking behavior and on binge-like eating of a highly preferred chocolate diet. Food seeking was measured in rats trained to respond for chocolate under a second-order schedule of reinforcement, in which prolonged periods of food-seeking behavior were maintained by contingent presentation of a reward-associated conditioned reinforcer. After reaching a stable baseline in both procedures, animals were treated with GSKI52I498 (0.I, I, and $3 \mathrm{mg} / \mathrm{kg} ; \mathrm{IP})$ or naltrexone (NTX, 0.I, I, and $3 \mathrm{mg} / \mathrm{kg}$; SC). The binge eating model was characterized by four temporally contiguous phases: I-h chow access, 2-h food deprivation, 10-min chow access, and I0-min access to either chocolate-flavoured food or standard chow. During training the rats developed binge-like hyperphagia of palatable food and anticipatory chow hypophagia (anticipatory negative contrast). Both compounds reduced binge-like palatable food hyperphagia. However, GSK I52 1498 reduced the impact of high hedonic value on ingestion more specifically than NTX, abolishing anticipatory chow hypophagia. GSKI52I498 also dose-dependently reduced food seeking both before and after food ingestion, whereas NTX reduced food seeking only after food ingestion. Thus, while both drugs affected the hedonic value of the preferred food, GSKI52I498 also directly decreased incentive motivation for chocolate. Selective $\mu$-opioid receptor antagonism by GSKI52I498 may have utility as a treatment for reducing maladaptive, palatability-driven eating behavior by reducing the motivational properties of stimuli that elicit the binge eating commonly associated with obesity.

Neuropsychopharmacology (2012) 37, 2643-2652; doi:I0.1038/npp.2012.128; published online I8 July 2012
\end{abstract}

Keywords: binge eating disorder; obesity; food seeking; conditioned stimulus; naltrexone; $\mu$-opioid receptor antagonist

\section{INTRODUCTION}

Obesity may be viewed as the product of a maladaptive interaction between physiological, psychological, and environmental factors that regulate palatable food intake by humans and animals (Bodnar, 2004; Zhang et al, 1998). Binge eating and increased responses to food-associated environmental stimuli are frequently associated with obesity.

Abundant evidence implicates the brain opioid systems in regulating food intake and in mediating the rewarding impact of palatable food intake (for a review see Nathan and Bullmore, 2009). Opioid receptor agonists increase, whereas

*Correspondence: Dr C Giuliano, Behavioral and Clinical Neuroscience Institute and Department of Experimental Psychology, University of Cambridge, Downing Street, Cambridge CB2 3EB, UK. Tel: + 4401223 765292, Fax: + 4401223722564 ,

E-mail: cg45।@cam.ac.uk

Received 23 March 2012; revised 7 June 2012; accepted 25 June 2012 opioid receptor antagonists decrease, food intake (Echo et al, 2002; Smith and Berridge, 2007; Will et al, 2003; Yeomans and Gray, 1997). $\mu$-Opioid receptors have a fundamental role in mediating hedonic responses to palatable foods with $\mu$-opioid receptor 'hotspots' having been identified in the pallidum and nucleus accumbens (NAcc), activation of which can increase hedonic reactions to sweet tastes (Peciña and Berridge, 2005), the intake of palatable food with a high sugar and high fat content (Kelley, 2004), as well as the consumption of the more preferred flavoured food when presented simultaneously with a less preferred food (Woolley et al, 2006). Conversely, in rats, antagonists at $\mu$-opioid receptors reduce (i) positive taste reactions (Shin et al, 2010), (ii) the intake of preferred $v s$ nonpreferred diets (Glass et al, 1996), (iii) saccharin consumption, and (iv) the preference for saccharin solutions over water (Lynch, 1986). The $\mu$-opioid antagonists also suppress subjective ratings of food palatability in humans (Fantino et al, 1986; Bertino et al, 1991; Drewnowski et al, 1992; Yeomans and Gray, 1997; Nathan et al, 2012). 
However, there is much less consensus concerning opioid mechanisms in incentive motivational processes that underlie the propensity to seek palatable foods. It has been shown that naloxone, a general opioid receptor antagonist with some selectivity toward $\mu$-opioid receptors, reduced the breakpoint, an index of the reinforcing value of the reward (Stoops, 2008), under a progressive ratio (PR) schedule for food (Cleary et al, 1996); whereas DAMGO, a $\mu$-opioid receptor agonist, infused into the NAcc increased it (Zhang et al, 2003). Mice with deletions of the $\mu$-opioid receptor showed lower levels of food-driven operant behavior measured under a PR schedule (Papaleo et al, 2007). These studies suggest that endogenous opioids may be involved in motivation, but do not fully distinguish between primary motivational effects and those that are secondary to changes in food value, or hedonic impact, following $\mu$-opioid receptor agonist or antagonist treatments.

In this study, therefore, we have investigated the behavioral effects of a novel, selective $\mu$-opioid receptor antagonist GSK1521498 (fpKi=9.24) with threefold higher affinity for the $\mu$-opioid receptor and no partial agonist action compared with naltrexone (NTX) (Ignar et al, 2011). We used both a food-seeking procedure and a binge-eating paradigm to test the hypothesis that $\mu$-opioid transmission is involved in food cue-elicited food-seeking behavior, as well as in palatability and associated binge eating.

To study the motivation to seek highly palatable food, we utilized a second-order schedule of chocolate pellet reinforcement in which a conditioned stimulus (CS) associated with chocolate ingestion supports high levels of instrumental-seeking behavior over delays to the delivery of a large chocolate reward. This procedure measures the motivation for the opportunity to binge on a palatable food, as well as the impact of ingestion of that food has on subsequent food seeking (Everitt et al, 1987). To investigate binge eating, we adapted the procedure established by Cottone et al (2008) in which emphasis is placed on the qualitative aspects of dietary restraint, mimicking the attempted abstinence of binge eaters from 'forbidden' food (Corwin, 2006). In this procedure, access to palatable food (chocolate) is brief (10 min), promoting rapid consumption of a large amount of chocolate, but in addition animals also self-restrict their intake of otherwise acceptable food (regular chow) in anticipation of access to the palatable chocolate (an 'anticipatory negative contrast' effect; Cottone et al, 2008). We show that $\mu$-opioid receptor antagonism reduces both the propensity to seek, and binge eating of, high incentive chocolate.

The data suggest the potential therapeutic efficacy of GSK1521498 in the treatment of maladaptive reward-driven eating behavior, binge eating, and obesity.

\section{MATERIALS AND METHODS}

\section{Animals}

Adult male and female Lister Hooded rats (Charles River, Margate, UK) weighing 280-320 $\mathrm{g}$ at the beginning of the experiments were individually housed under a reversed $12 \mathrm{~h}$ light/dark cycle (lights off at 0800 hours). Rats had access to corn-based rodent chow and water ad libitum for the entire period of the experiments (binge eating experiments) and after a few weeks of food restriction with $18 \mathrm{~g} / \mathrm{day}$ lab chow, sufficient to maintain body weight (food seeking experiments). Experiments were performed between 07.45 and 16.00 hours, 6-7 days a week and they were conducted according to the UK (1986) Animal (Scientific Procedures) Act (Project license 80/2234) and the GSK Policy on the Care, Welfare and Treatment of Laboratory Animals.

\section{Drugs}

GSK1521498 was supplied by GlaxoSmithKline (Harlow, UK) in an aqueous stock solution of $4 \mathrm{mg} / \mathrm{ml}$, expressed as free base, in an acidified hydroxypropyl beta-cyclodextrincontaining vehicle. Before administration, the stock solution was diluted with a phosphate buffer $(\mathrm{pH} \sim 6.5)$ to yield a 1 or $0.1 \mathrm{mg} / \mathrm{ml}$ solution. The formulation components were optimized to yield a final solution $\mathrm{pH}$ in the range of $5.2-6.5$, and an osmolality of $\sim 290 \mathrm{mOsm} / \mathrm{kg}$. The diluted solution was filtered through a 0.22 -micron filter before parenteral administration. The vehicle solution, the composition of which was identical to the GSK1521498 stock solution save for omission of the drug substance, was also supplied by GlaxoSmithKline and diluted with phosphate buffer to yield a final solution $\mathrm{pH}$ in the range of 5.2-6.5. Naltrexone (NTX; Sigma, UK) was dissolved in sterile physiological saline to yield 3,1 , or $0.1 \mathrm{mg} / \mathrm{ml}$ solution. Drug doses, time before testing and administration route were based on previous studies (Ignar et al, 2011; Williams and Broadbridge, 2009; see Supplementary Figure S1). All the solutions were prepared freshly on each test day and in all studies the different doses used were administered in a counterbalanced order following a Latin Square design. Two or three days of drug washout and re-baselining of behavior separated each treatment.

\section{Experimental Procedures}

A preliminary food preference study was run with female $(n=6)$ and male rats $(n=6)$. The same animals were tested on food seeking and taking procedures. A new group of animals ( $n=12$ females) was tested in the binge eating procedure. An additional group of animals ( $n=9$ per group, males) was tested in the conditioned place preferencel aversion procedure.

\section{Preference for Chocolate Pellets Over Chow}

Acclimated rats ( $n=6$ females and $n=6$ males) were provided with concurrent access for 15 consecutive days in the home cage to chow diet (Harlan-Teklad Global 18\% protein diet: $44.2 \%$ (kcal) carbohydrate, $6.2 \%$ fat, 18.6\% protein; metabolizable energy $3.10 \mathrm{kcal} / \mathrm{g}$ ) and to chocolateflavoured pellets (chocolate-flavoured formula PJPPP: 69.1\% (kcal) carbohydrate, $11.8 \%$ fat, $19.1 \%$ protein, metabolizable energy $3.70 \mathrm{kcal} / \mathrm{g}$; formulated as $45-\mathrm{mg}$ precision food pellets, Research Diets, New Brunswick, NJ).

Every $24 \mathrm{~h}$ chow and chocolate pellets were weighed. Food preference was calculated as the percentage of total 24-h (kcal) intake consumed in the form of the chocolateflavoured pellets. 


\section{Food Seeking and Taking Procedure}

The same animals used for the food-preference experiment ( $n=6$ females and $n=6$ males) were used for this experiment. They were only initially food restricted, to facilitate the acquisition of the task. Behavioral training was conducted in operant chambers (Med Associates, St Albans, VT) equipped with two retractable levers, a pellet receptacle between the levers connected with a pellet dispenser, a cue light above each lever, and a white house light. Active and inactive levers were counterbalanced between left and right sides. The first step was to train rats to respond on a lever for chocolate pellets under a fixed ratio 1 (FR1) schedule of reinforcement. Responses on the inactive lever had no programmed consequences but were recorded to assess discriminated responding and general levels of motor activity. Each lever press resulted in a chocolate-flavoured pellet delivery, illumination of a CS light above the active lever for $20 \mathrm{~s}$, retraction of both levers, and extinction of the house light for $20 \mathrm{~s}$ (time out, TO). After this TO, the house light was again illuminated, CS was extinguished, and the two levers were again inserted into the chamber. Rats were limited to a maximum of 30 pellets per 2-h session. A fixed interval (FI) schedule of reinforcement was introduced as the second step of the training. The FI increased daily from FI1 min, to FI2, FI4, FI8, and FI10 min before stabilizing at FI $15 \mathrm{~min}$ for three consecutive sessions. Finally, a secondorder schedule of reinforcement was introduced, in which every 10th active lever press resulted in a brief CS presentation for $1 \mathrm{~s}$ (FI15(FR10:S)); following the tenth active lever press after the FI15 min had elapsed, chocolateflavoured pellets were delivered and the CS was presented for $20 \mathrm{~s}$. The number of chocolate pellets delivered at the end of the fixed interval progressively increased from 2 to 15 under each FI stage, and finally 20 pellets were delivered on conclusion of each trial of the second-order schedule of reinforcement. Sessions terminated after either completion after two earned 20-pellet-reward deliveries, or $40 \mathrm{~min}$, whichever criterion was met first.

\section{Experiments 1A and 1B: Effects of GSK1521498 and Naltrexone on Food Seeking Under a Second-Order Schedule of Reinforcement}

Following extensive training under the second-order schedule ( $>1$ month), animals were treated with GSK1521498 0, 0.1, 1 , and $3 \mathrm{mg} / \mathrm{kg}$ (IP) $30 \mathrm{~min}$ before the session or with NTX 0 , $0.1,1$, and $3 \mathrm{mg} / \mathrm{kg}$ (SC) $10 \mathrm{~min}$ before the session, in a between subject counterbalanced design.

\section{Experiments 2A and 2B: Effects of GSK1521498 and Naltrexone on Food Taking Under Continuous Reinforcement}

The same animals trained to seek highly palatable food under the second-order schedule of reinforcement were stabilized in responding under a FR1 schedule of reinforcement. Animals were treated with GSK1521498 0, 0.1, 1, and $3 \mathrm{mg} / \mathrm{kg}$ (IP) $30 \mathrm{~min}$ before the session or with NTX $0,0.1,1$, and $3 \mathrm{mg} / \mathrm{kg}$ (SC) $10 \mathrm{~min}$ before the session, in a between subject counterbalanced design.

\section{Feeding and Binge Eating Procedure}

Naive rats acclimatized to the animal facility ( $n=12$ females) were used for this set of experiments. Animals were never food deprived. During the course of the experiment, one animal was excluded from the analysis due to ill health.

Baseline feeding. Every day $15 \mathrm{~min}$ before dark cycle onset, the animals were transferred to a room, weighed, and placed individually in testing chambers (Med Associates) equipped with two food intake monitors with retractable doors and a precision sensor to continuously monitor the weight of the food cup to within $0.01 \mathrm{~g}$, a white house light, and bottles of water. Each test session consisted of four contiguous periods in the following order: (a) 1-h chow access, (b) 2-h food deprivation, (c) 10-min access to a chow feeder, and (d) 10-min access to a different chow feeder. Rats were then rapidly returned to the home cage with chow available ad libitum. Post-session daily home-cage chow intake (in $20 \mathrm{~h}$ $40 \mathrm{~min}$ ) was also measured. Water was always available ad libitum. Food was weighed with $0.01 \mathrm{~g}$ precision to measure caloric intake during the session and during the day, when the animals were in the home cage. Over $\sim 3$ weeks, intake from the 10-min feeders stabilized.

Binge eating paradigm. Rats, matched for daily food intake and food intake within each test session period, were divided into two groups: the 'chow/chow' control group, which received chow access from both 10-min feeders, and the 'chow/chocolate' binge group, which received chow in the first 10-min feeder and chocolate-flavoured pellets in the second 10-min feeder. Rats were tested daily.

\section{Experiments 3A and 3B: Effects of GSK1521498 and Naltrexone on Binge Eating Paradigm}

After 15 days of chow/chocolate exposure at the second feeder, rats ( $n=11$ females) received GSK1521498 0, 0.1, 1 , and $3 \mathrm{mg} / \mathrm{kg}$ (IP) $30 \mathrm{~min}$ before the first 10-min chow access or NTX $0,0.1,1$, and $3 \mathrm{mg} / \mathrm{kg}$ (SC) $30 \mathrm{~min}$ before the first 10 -min chow access.

\section{Conditioned Place Preference/Aversion Procedure}

Naive animals ( $n=9$ per group, males) were used for this experiment. All behavioral training and testing took place in a Y-maze apparatus (Med Associates) consisting of three chambers identical in size $(38.1 \times 38.1 \times 20 \mathrm{~cm})$, two of them separated by manually operated guillotine doors from a smaller central, white PVC floored compartment in the shape of an equilateral triangle (side $38.1 \mathrm{~cm}$ ) (Ito et al, 2006). The two chambers were distinguished by specific physical features: a stainless steel grid rod floor consisting of $3 / 16^{\prime}(4.8 \mathrm{~mm})$ rods $v s$ a stainless steel tray, and walls with painted stripes $v s$ painted dots. The floor and walls of the apparatus were wiped down with water following each session to eliminate any odor traces.

The protocol consisted of habituation (2 days), conditioning (2 days), and test. During the habituation days, baseline preferences were assessed by placing rats in the central compartment of the apparatus and allowing free access to all compartments for $15 \mathrm{~min}$. During the conditioning 
phase, the rats were injected with GSK1521498 or NTX and confined in one of the compartments for $30 \mathrm{~min}$. The conditioned side was arbitrarily assigned to each rat, with the groups being matched so that times spent in the to-be-conditioned compartment during the second session of habituation were equal. The treatment orders of injection (drug or vehicle) and of compartment were counterbalanced across subjects. Conditioning sessions were conducted once daily. During the test day, the rats were placed in the central compartment and the time spent in each compartment was recorded.

\section{Experiments 4A and 4B: Motivational Effects of GSK1521498 and Naltrexone Under Conditioned Place Preference/Aversion Procedure}

During the conditioning phase, the rats were injected with GSK1521498 0, 1, and $3 \mathrm{mg} / \mathrm{kg}$ (IP) $30 \mathrm{~min}$ before the session or NTX 0,1 , and $3 \mathrm{mg} / \mathrm{kg}$ (SC) $10 \mathrm{~min}$ before the session and confined in one of the compartments for $30 \mathrm{~min}$. On the test day, the animals were not injected, placed in the central compartment, and were given free access to the entire chamber for $15 \mathrm{~min}$. The amount of time spent in each compartment was recorded.

\section{Statistical Analysis}

For binge eating experiments, intakes (kcal) during each period of the test session and in the home cage were analyzed using repeated measures analysis of variance (ANOVA) (SPSS 19, Chicago, IL) with dose as the withinsubjects factor and diet history as a between-subjects factor.

For food seeking experiments, active and inactive lever responses during the first and second 15-min intervals were analyzed using repeated measures ANOVA (SPSS 19) with dose as the within-subject factor. For food self-administration experiments, food deliveries per session were analyzed.

For all analyses, upon confirmation of significant main effects, differences among individual treatments were analysed using the Sidak post hoc test. Statistical significance was set at $p<0.05$. Student's $t$-tests (two-tailed) were used to compare means of the experimental groups in the conditioned place preference/aversion experiment.

\section{RESULTS}

\section{Preference for Chocolate Pellets Over Chow}

All the rats receiving concurrent ad libitum access to chow diet and to iso-caloric chocolate-flavoured pellets in the home-cage for 15 consecutive days showed preference for chocolate-flavoured pellets (males: $74.93 \pm 9.57 \%$ vs females: $88.58 \pm 3.46 \%$ ) on the first day, and there were no differences between males and females $(t(10)=1.34$; NS). However, starting from the second day, female rats showed a clear preference for chocolate-flavoured pellets while male rats did not (repeated measures ANOVA revealed a main effect of time: $(\mathrm{F}(13,130)=2.6 ; p=0.003)$ and a timeXsex interaction: $(F(13,130)=1.8 ; p=0.051$; Figure 1a). Moreover, there was no difference in chocolate and chow intake from day 2 to 15 in males $(t(5)=0.59$; NS), but there was an increase in chocolate compared with chow intake in females $(t(5)=3.51 ; p=0.017$; Figure $1 \mathrm{~b})$.

\section{Food Seeking and Taking Procedure}

Experiment 1a: effect of GSK1521498 on food seeking under a second-order schedule of reinforcement. In total, 12 rats, 6 males and 6 females, acquired responding for highly palatable food under a second-order schedule of reinforcement, both genders making on average 500 responses during the first $15 \mathrm{~min}$ before the first food delivery (mean \pm SEM: $566.94 \pm 91.52$ for males $v s$ $457.28 \pm 56.78$ for females, $t(10)=1.02$, NS). Treatment with GSK1521498 resulted in a significant dose-dependent decrease in responding (main effect of dose $(\mathrm{F}(3,30)=17.7 ; p<0.0001)$ ) and a doseXsex interaction $(F(3,30)=4.8 ; p=0.008)$ in the first (pre-ingestive) 15-min interval of the session. After reward delivery, all rats showed a dose-dependent decrease in responding $(\mathrm{F}(3,30)=17.6 ; p<0.001)$. Post hoc analysis revealed a significant decrease in food seeking in males treated with 1 and $3 \mathrm{mg} / \mathrm{kg}$ of GSK1521498, whereas in females only at $3 \mathrm{mg} / \mathrm{kg}$ during the first interval of the session. In all animals there was a decrease in responding during the post-ingestive second interval of the session at both 1 and $3 \mathrm{mg} / \mathrm{kg}$ GSK1521498 (Figure 2).

Experiment 1b: effect of naltrexone on food seeking under a second-order schedule of reinforcement. In both males
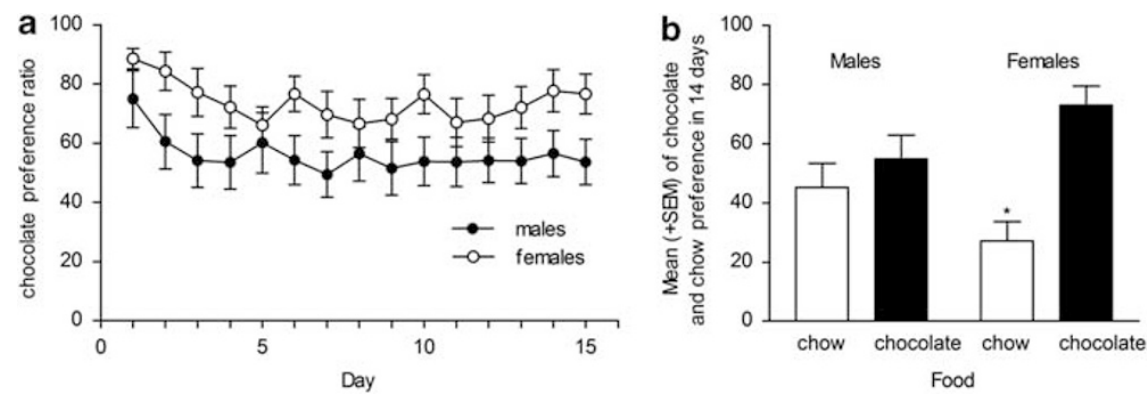

Figure I Food preference. Male $(n=6)$ and female $(n=6)$ rats were provided with concurrent access to chow diet and to chocolate-flavoured pellets in the home-cage for 15 consecutive days. Chow and chocolate pellets left in the home-cage were weighed every 24-h. Data are expressed as the averaged \% of total 24-h (kcal) intake ( \pm SEM) consumed in the form of the chocolate-flavoured pellets by females (open circles) and males (closed circles) (a). On the right, the first two bars show the means of chow (white) and chocolate (black) preference over I4 days in males. The second set of two bars show the means of chow (white) and chocolate (black) preference over 14 days in females (b). $* p<0.05$ compared with chocolate preference. 

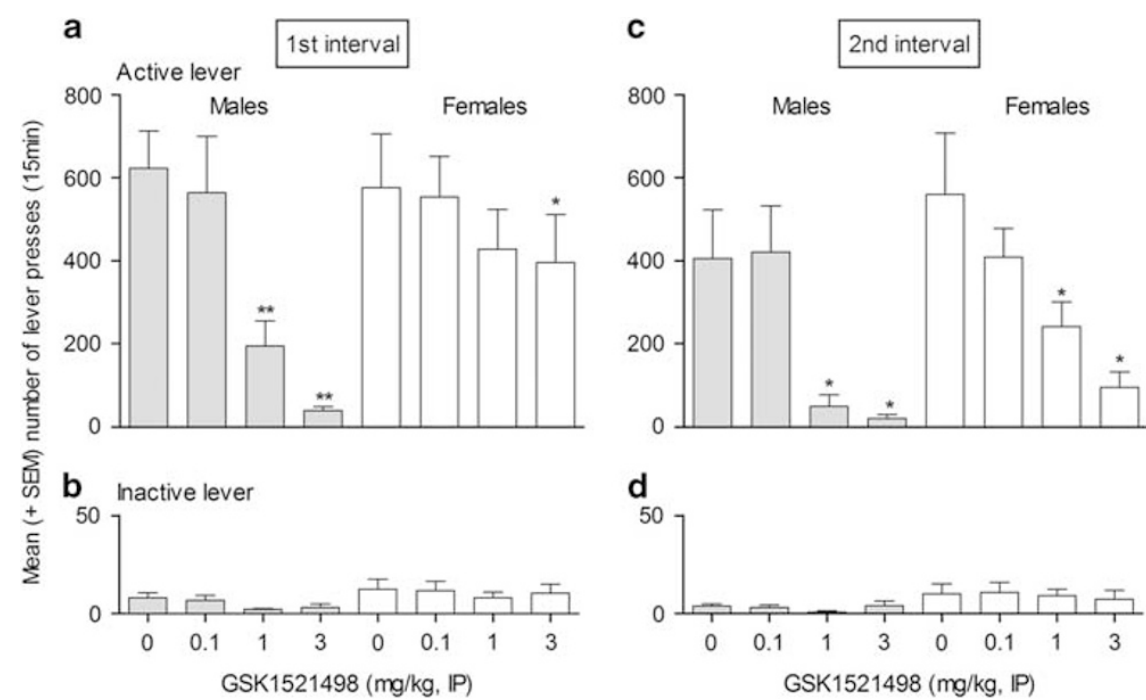

Figure 2 Effect of GSKI52I498 on food seeking. Effects of GSK I52 498 on food seeking under a second-order schedule of reinforcement during the first food-free interval $(a, b)$ and after food delivery (second interval) $(c, d)$ in male $(n=6$, gray bars) and in female $(n=6$, white bars) rats. Data shown are mean $(+$ SEM) number of presses on the active $(a, c)$ and inactive lever $(b, d) . * p<0.05$, *** $p<0.01$ compared with vehicle-treated animals.
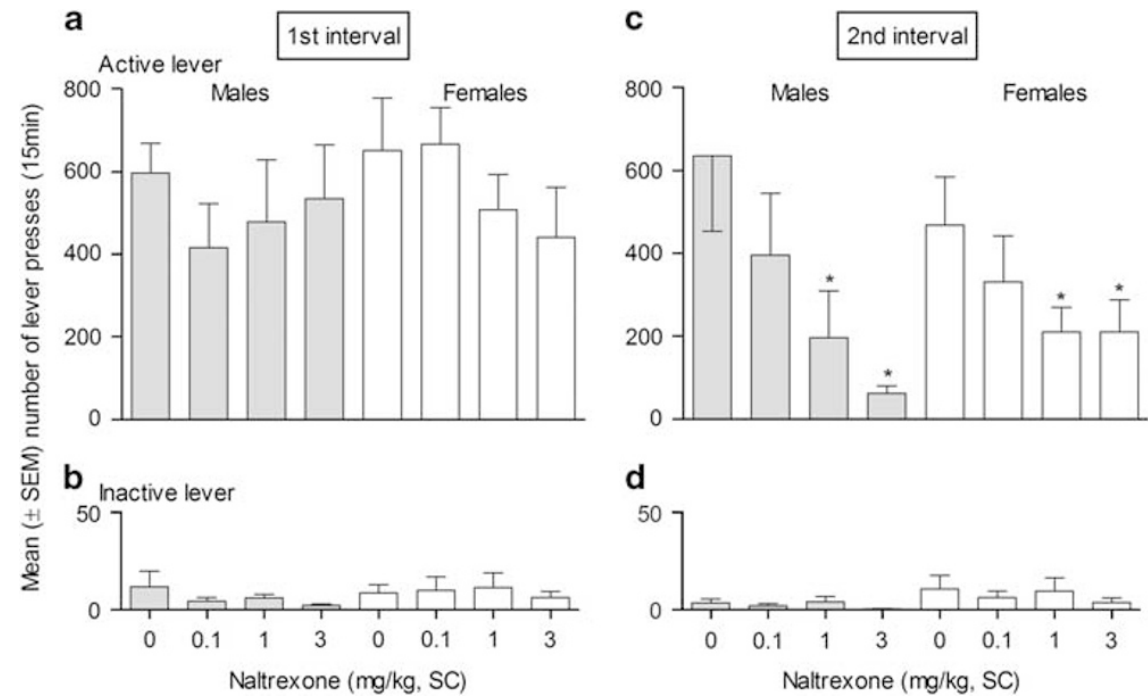

Figure 3 Effect of naltrexone (NTX) on food seeking. Effects of NTX on food-seeking under a second-order schedule of reinforcement during the first food-free interval $(a, b)$ and after food delivery (second interval) $(c, d)$ in male $(n=6$, gray bars) and in female $(n=6$, white bars) rats. Data shown are mean $( \pm \mathrm{SEM})$ number of presses on the active $(\mathrm{a}, \mathrm{c})$ and inactive lever $(\mathrm{b}, \mathrm{d}) .{ }^{*} p<0.05$ compared with vehicle-treated animals.

and females, treatment with NTX was without effect on responding during the first interval of the session (no main effect of dose: $(\mathrm{F}(3,30)=1.3$; NS); no main effect of sex: $(F(3,30)=0.9$; NS)). After chocolate pellets delivery in the second interval, NTX-treated subjects had decreased responding in a dose-dependent manner $(F(3,30)=6.7$; $p=0.001)$. Post hoc analysis revealed a significant decrease in food seeking after treatment with $0.1,1$, and $3 \mathrm{mg} / \mathrm{kg}$ during the post-ingestive second interval of the session in both males and females (Figure 3).

Experiment 2a: effect of GSK1521498 on responding for food under continuous reinforcement. The same animals trained to seek highly palatable food under a second-order schedule of reinforcement were stabilized to respond under a simple FR1 schedule of reinforcement. GSK1521498 decreased the taking response for food under a FR1 schedule. GSK1521498 reduced the number of food pellets eaten by the end of the session (most of the animals left in the food magazine some of the food pellets earned by pressing the active lever; main effect of dose: $(\mathrm{F}(2,20)=38 ; p<0.001)$; a doseXsex interaction: $(\mathrm{F}(2,20)=26.6 ; p<0.001)$; and main effect of sex: $(\mathrm{F}(1,10)=26.6 ; p<0.001$; Supplementary Figure S2A).

Experiment 2b: effect of naltrexone on responding for food under continuous reinforcement. NTX affected responding for food under a FR1 schedule, reducing the number of food pellets eaten by the end of the session (main effect of dose: 
$(\mathrm{F}(2,20)=5.6 ; p=0.007)$; doseXsex interaction: $(\mathrm{F}(2,20)=4.7$; $p=0.021)$; and main effect of sex: $(\mathrm{F}(1,10)=5.9 ; p=0.036))$. Post hoc analysis revealed a significant decrease in food taking in male rats after treatment with NTX $3 \mathrm{mg} / \mathrm{kg}$ $(p<0.05$; Supplementary Figure S2B).

\section{Feeding Procedure}

Binge eating. As only female rats developed chocolate preference, the binge eating study was conducted on females, as previously also described by Cottone et al (2008). Rats receiving highly limited access to the preferred diet (chow/ chocolate) developed chow hypophagia (an 'anticipatory negative contrast' effect) from the first feeder (main effect of diet history: $(\mathrm{F}(1,9)=11.3 ; p=0.008)$ and main effect of time: $(\mathrm{F}(14,126)=2.5 ; p=0.007))$ and hyperphagia of the preferred diet from the second feeder (main effect of diet history: $(\mathrm{F}(1,9)=203.2 ; p<0.001)$, main effect of time: $(\mathrm{F}(14,126)=8.1$; $p<0.001)$, and a timeXdiet history interaction: $(\mathrm{F}(14,126)=$ 9.3; $p<0.001)$ ). In addition, home-cage chow intake of chow/chocolate-fed rats was lower than that of chow/chowfed rats (main effect diet history: $(\mathrm{F}(1,9)=17.1 ; p=0.003)$; Figure 4).

\section{Experiment 3A: Effect of GSK1521498 on Binge Eating}

Under the vehicle condition, chow/chocolate-fed rats showed anticipatory chow hypophagia and chocolate pellet hyperphagia. Treatment with GSK1521498 differentially affected the first 10-min chow intake according to diet history (main effect of diet history: $(\mathrm{F}(1,9)=5.5 ; p=0.043)$ and a diet historyXdose interaction: $(\mathrm{F}(3,27)=6.1 ; p=0.006))$. Thus, GSK1521498 treatment resulted in increased chow intake in chow/chocolate-fed rats and decreased chow intake in chow/chow-fed rats in a dose-dependent manner, ie, it abolished anticipatory chow hypophagia. Post hoc analysis revealed a significant increase in chow intake in chow/chocolate-fed rats treated with $3 \mathrm{mg} / \mathrm{kg}$ GSK1521498 $(p<0.05)$. GSK1521498 also differentially affected the second 10 -min chocolate intake according to diet history (main effect of diet history: $(\mathrm{F}(1,9)=508.2 ; p<0.001)$ and a diet historyXdose interaction: $(\mathrm{F}(3,27)=3.1 ; p=0.043)$. Thus, GSK1521498 treatment dose dependently resulted in a decreased chocolate intake in chow/chocolate-fed rats (main effect of dose: $(F(3,27)=$ $3.2 ; p=0.038)$ ). Post hoc analysis revealed a significant decrease in chocolate intake from the second chocolate feeder at the $1 \mathrm{mg} / \mathrm{kg}(p<0.05)$ and $3 \mathrm{mg} / \mathrm{kg}(p<0.05)$ doses of GSK1521498. GSK1521498 also differentially decreased chow intake in the home-cage according to diet history (main effect of dose: $(\mathrm{F}(3,27)=31.4 ; p<0.001)$ and main effect of diet history: $(\mathrm{F}(1,9)=27.5 ; p=0.001)$ ). Post hoc analysis revealed a significant decrease in home-cage chow intake in chow/chocolate-fed rats treated with GSK1521498 $1 \mathrm{mg} / \mathrm{kg}(p<0.05)$ and $3 \mathrm{mg} / \mathrm{kg}(p=0.001)$ and also in chow $/$ chow-fed rats treated with GSK1521498 $3 \mathrm{mg} / \mathrm{kg}(p=0.002$; Figures $5 \mathrm{a}-\mathrm{c}$ ).

\section{Experiment 3B: Effect of Naltrexone on Binge Eating}

Under vehicle condition, chow/chocolate-fed rats showed (as explained above) anticipatory chow hypophagia and chocolate pellet hyperphagia. NTX treatment had no significant effect on the first 10-min chow intake (main effect of dose: $(\mathrm{F}(3,27)=0.5 ; \mathrm{NS}))$, ie, it did not affect anticipatory negative contrast. However, NTX differentially affected the second 10-min intake according to diet history, reducing the chocolate intake in chow/chocolate-fed rats without altering chow intake in chow/chow-fed rats (main effect of dose: $(\mathrm{F}(3,27)=4.7 ; p=0.010)$, main effect of diet history: $(\mathrm{F}(1,9)=67.6 ; p<0.001)$ and a diet historyXdose interaction: $(\mathrm{F}(3,27)=5.9 ; p=0.003))$. Post hoc analysis revealed a significant decrease in the second 10-min chocolate intake at $1 \mathrm{mg} / \mathrm{kg}(p<0.05)$ and $3 \mathrm{mg} / \mathrm{kg}(p<0.01)$ doses of NTX. The animals differentially maintained chow intake in the
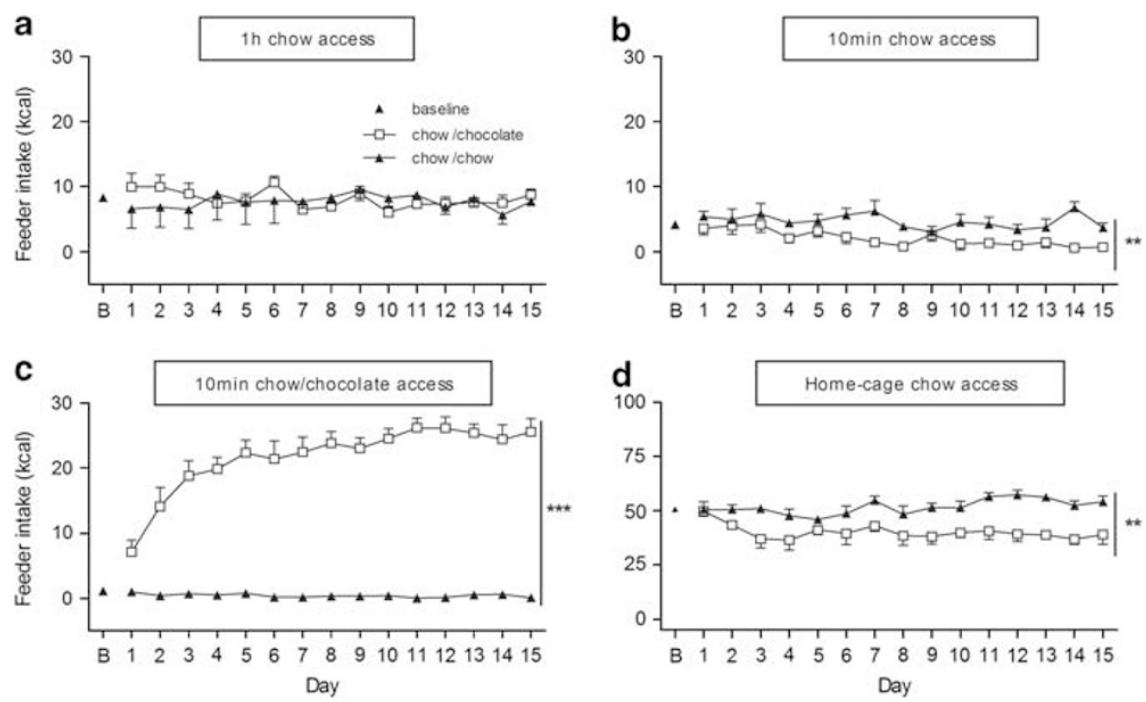

Figure 4 Binge eating paradigm. The experimental session was divided into four contiguous phases: I-h chow access (a), 2-h food deprivation, I0-min access to chow (b), and I0-min access to chow or chocolate (c). Chow intake in the home-cage, after the experimental session, is also shown (d). Values represent the averaged intakes (kcal) from days I-I5 ( \pm SEM), after acquisition of a stable baseline, in chow/chow-fed $(n=5$ females, open squares) and in chow/chocolate-fed animals ( $n=6$ females, closed squares). $* * *<0.01$, **** $p<0.001$ compared with chow/chow-fed animals. 

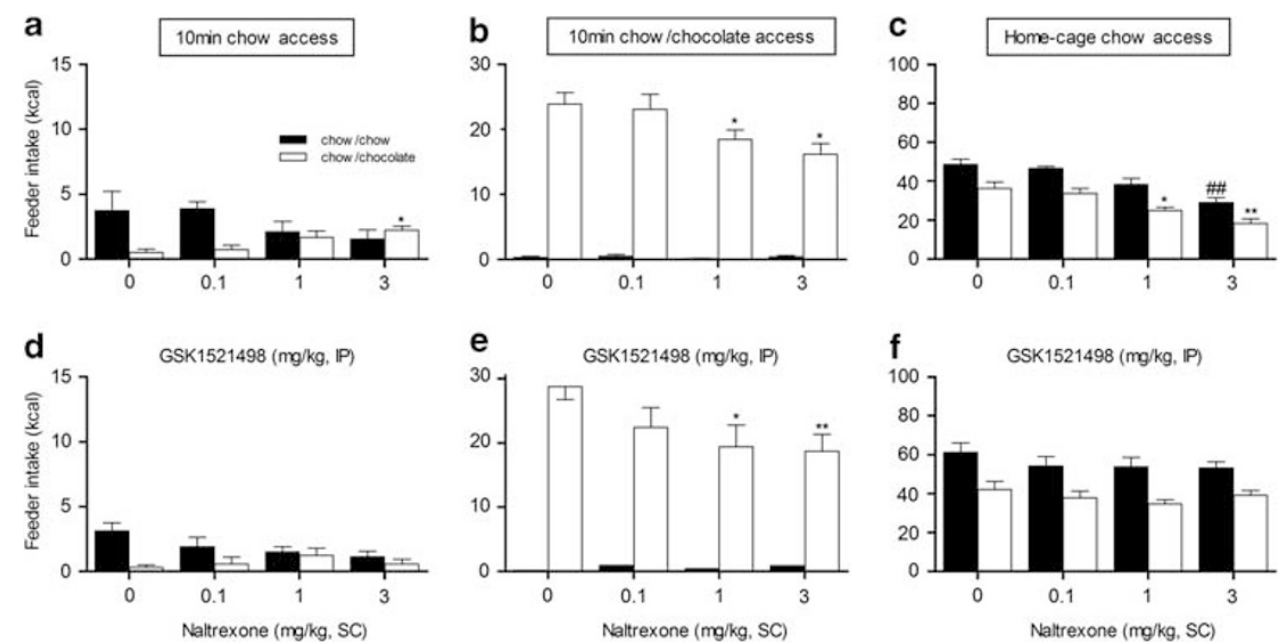

Figure 5 Effects of GSKI52I498 and naltrexone (NTX) on binge eating. On the top, effect of GSKI5I2I498 on I0-min access to chow (a), I0-min access to chow or chocolate (b), access to chow in home-cage, and after the experimental session (c). Values represent the averaged caloric intakes (kcal) $( \pm$ SEM) in chow/chow-fed animals $(n=5$ females, black bars) and in chow/chocolate-fed rats $(n=6$ females, white bars). $* p<0.05$, $* * *<0.01$ compared with vehicle-treated chow/chocolate-fed animals; ${ }^{\# \#} p<0.0$ l compared with vehicle-treated chow/chow-fed animals. On the bottom, effect of NTX on I0-min access to chow (d), I0-min access to chow or chocolate (e), access to chow in home-cage, and after the experimental session ( $f$ ). Values represent the averaged caloric intakes (kcal) $\left( \pm\right.$ SEM) in chow/chow-fed animals $\left(n=5\right.$, black bars) and in chow/chocolate-fed rats $\left(n=6\right.$, white bars). ${ }^{*} p<0.05$, *** $p<0.0$ I compared with vehicle-treated chow/chocolate-fed animals.

home-cage according to diet history, ie, home-cage chow intake of chow/chocolate-fed rats was lower than that of chow/chow-fed rats (main effect of diet history: $(\mathrm{F}(1,9)=$ 18.3; $p=0.002)$ ), but this was not affected by NTX (both the main effect of dose $(\mathrm{F}(3,27)=2.3$; NS), and the diet historyXdose interaction $(\mathrm{F}(3,27)=0.4 ; \mathrm{NS})$ were not significant; Figures 5d-f).

\section{Experiments 4A and 4B: Motivational Effects of GSK1521498 and Naltrexone Under Conditioned Place Preference/Aversion Procedure}

There was no evidence for significant conditioned place aversion or preference following GSK1521498 or NTX. Specifically, there was no difference between time spent in the vehicle- and drug-paired chambers either following treatment with GSK $15214981 \mathrm{mg} / \mathrm{kg} \mathrm{i}(t(16)=0.56$; NS) or $3 \mathrm{mg} / \mathrm{kg}(t(16)=0.92 ; \mathrm{NS})$, or following treatment with NTX $1 \mathrm{mg} / \mathrm{kg}(t(16)=0.41 ; \mathrm{NS})$ or $3 \mathrm{mg} / \mathrm{kg} \quad(t(16)=1.15 ; \mathrm{NS}$; Supplementary Figure S3).

\section{DISCUSSION}

This study describes the effects of GSK1521498, a novel, selective $\mu$-opioid receptor antagonist, and NTX, a $\mu$-opioid receptor antagonist, on two distinct aspects of eating behavior: food seeking and binge-like eating. Both GSK1521498 and NTX reduced food seeking after ingestion of palatable food, indicating an effect mediated by a reduction in the hedonic value of the food. However, only GSK1521498 reduced the seeking response for chocolate before its delivery for ingestion, suggesting an additional effect on incentive motivational mechanisms controlling food seeking. Both GSK1521498 and NTX reduced binge-like palatable food hyperphagia, but GSK1521498 also exerted a more specific effect on the impact of the hedonic value of the food and intake than did NTX, reducing the anticipatory chow hypophagia before the highly palatable food was available for ingestion. Neither GSK1521498 nor NTX resulted in a conditioned place aversion under the same dosing regimens used in the binge eating and food-seeking procedures, indicating that a drug-induced negative affective state does not account for reductions in eating or instrumental responding.

\section{Food Seeking}

GSK1521498, but not NTX, markedly reduced the number of active lever presses during the first interval of the session, before food ingestion, suggesting an effect on incentive motivational mechanisms controlling food seeking and intake, or on the impact of the chocolate-associated conditioned reinforcer to maintain responding, as it is well known that environmental cues previously associated with food exert powerful control over food consumption that can override regulatory signals to stimulate eating even in sated states (Petrovich et al, 2007; Weingarten, 1983).

It is of note that GSK1521498 and NTX differentially affected male and female rats: the reduction in food seeking was greater in males than in females, perhaps reflecting that males preferred chocolate less than females (as revealed by the food preference study). Clinical data also show that alcohol- or drug-dependent men and women may respond differentially to NTX treatment, with men usually showing a better treatment outcome compared with women (Garbutt et al, 2005; Pettinati et al, 2008) in terms of a reduced tendency to relapse. This may reflect sex differences in pharmacokinetics, such as the distribution, metabolism, and elimination of medications (Gandhi et al, 2004), but it has also been suggested that there are gender differences in the sensitivity of the endogenous opioid system to opioid receptor agents (O’Malley et al, 2000). 
There are several putative neural sites at which $\mu$-opioid receptor antagonism may cause decreases in the propensity to seek food. Dopaminergic transmission in the NAcc has a major role in incentive motivational processing for food (Smith and Berridge, 2007). $\mu$-Opioid receptors localized on the GABAergic interneurons in the VTA provide one site at which GSK1521498 might act to decrease dopamine release in the NAcc to reduce food seeking (Johnson and North, 1992; Yun et al, 2004).

In addition, within the basolateral amygdala (Le Merrer et al, 2009) $\mu$-opioid receptors have been shown to be necessary for encoding the incentive value of ingestive rewards, but not their palatability (Wassum et al, 2009). By contrast, $\mu$-opioid mechanisms in the ventral pallidum have clearly been shown to mediate 'hedonic' responses, in particular, the attribution of pleasure value to the food (Peciña and Berridge, 2005; Smith and Berridge, 2005, 2007). Both compounds reduced food seeking after chocolate ingestion and responding for food under continuous reinforcement, suggesting that reducing the hedonic value of the ingested preferred food reduced the tendency to work for it, as might be expected (Balleine and Dickinson, 1992).

\section{Binge-Like Eating}

GSK1521498 was tested on binge-like eating in female rats, after a preliminary food preference study in which ad libitum access to chow and chocolate-flavoured pellets was provided to both female and male rats. Females showed a clear preference for chocolate pellets compared with males. These data confirm those in the human literature identifying chocolate as the single food that is most craved by women (Rozin et al, 1991). In fact, females tend to prefer snack-related comfort foods (defined as foods the consumption of which evokes a psychologically comfortable and pleasurable state for a person, such as candy and chocolate) compared with males who prefer more substantial, meal-related foods such as pizza, pasta, or beef (Wansink et al, 2003). Although it is well known that food intake fluctuates over the estrous cycle, and in women is lower during the peri-ovulatory period (Asarian and Geary, 2006), this variable was not a focus of this study as binge eating tends not to show cyclic variation or be affected by ovarian hormone treatment ( $\mathrm{Yu}$ et al, 2008, 2011). The brief 10-min periods of access to the palatable food used here to generate binge intake are likely insensitive to cyclic behavioral changes.

Binge eating is defined as the consumption of an unusually large amount of food, coupled with a sense of being out of control during a brief, defined period of time. These discrete episodes of rapid and excessive food consumption are not driven by hunger or metabolic need and for this reason highly palatable food is usually the object of these consummatory episodes. In this study, all animals developed binge-like hyperphagia of highly palatable food and anticipatory hypophagia of a less preferred alternative. This effect has been described as a form of 'anticipatory negative contrast' (Flaherty et al, 1995) and not simply homeostatic energy compensation, as there was no inverse correlation between the amount ingested from the first chow feeder and that ingested at the second feeder (Cottone et al, 2008). The chow/chocolate-fed rats also decreased ad libitum intake of the less-preferred chow in their home-cages, which might suggest a possible contribution of energy homeostatic mechanisms in home cage chow intake to compensate for the high chocolate intake by chow/chocolate-fed rats over the days of the experiment. Both GSK1521498 and NTX reduced binge-like palatable food hyperphagia, consistent with the hypothesis that inhibition of the endogenous opioid system, in particular that mediated by $\mu$-opioid receptors, influences hedonic mechanisms controlling food intake in humans and in animals (Olszewski and Levine, 2007). This has been confirmed in studies showing that stimulation of $\mu$-opioid receptors in the NAcc preferentially facilitates the intake of palatable food (Zhang et al, 1998), or that chronic suppression of $\mu$-opioid receptor signaling in the same area significantly reduces palatable food intake and the development of diet-induced obesity in rats (Lenard et al, 2010). Consistent with these data, opioid receptor antagonists, such as naloxone or nalmefene, have been shown completely to block binge eating in rats with a history of dieting and stress (Boggiano and Chandler, 2006) or with short-term access to palatable food as seen in this experiment (Cottone et al, 2008).

GSK1521498 exerted a greater effect on the impact of the hedonic value of the food on intake than did NTX, as it also reduced the anticipatory chow hypophagia seen before the highly palatable food was available for ingestion. It has previously been shown that opioid receptor antagonists (eg, nalmefene) increase the size of 'consummatory successive negative contrast' (Cottone et al, 2008), defined as a temporary reduction in responding to a smaller reward by animals previously exposed to a larger reward, compared with the responding observed in a control group always exposed to the smaller reward (Pellegrini et al, 2005). By analogy, here we confirm the involvement of the opioid system not only in hedonic mechanisms leading to consummatory behavior mediated, for example, by the proximal taste and smell of food, but also in the more distal, anticipatory incentive processes by which the animal comes to expect contact with palatable food and approaches it (Cottone et al, 2008; Pellegrini et al, 2005). Interestingly, GSK1521498 had potent effects on two distinct measures of food anticipation: anticipatory negative contrast and responding in the initial component of the food-seeking second-order schedule, perhaps suggesting a dopaminemediated mechanism in the prediction or anticipation of rewarded events (Schultz, 2002; Schultz et al, 1997). The differential potency of GSK1521498 compared with NTX is possibly due to its more selective action at $\mu$-opioid receptors and/or its specific action on learned associative and appetitive processes underlying food selection (Barbano and Cador, 2006). It has been shown that GSK1521498 is 14- and 20 -fold selective for human $\mu$-over $\kappa$ - and $\delta$-opioid receptors, and in rats is 52- and 66-fold selective for $\mu$ - over $\kappa$ - and $\delta$-opioid receptors, whereas NTX is 4 - and 10 -fold selective for human $\mu-v s \kappa$ - and $\delta$-opioid receptors, and 20 - and 25 -fold selective for rat $\mu$-compared with $\kappa$ - and $\delta$-opioid receptors (Ignar et al, 2011).

\section{Therapeutic Implications}

The results of this study show that GSK1521498, with its selective and more complete opioid receptor antagonist 
activity, may uniquely have the dual effect to reduce the motivation for, as well as the hedonic impact of, highly preferred food, thereby reducing ingestion. Drugs that inhibit $\mu$-opioid receptor signaling are reported to be effective across a range of disorders characterized by compulsive consumption of, for example, food, alcohol, opiates, or stimulant drugs. GSK1521498 is currently in clinical development for the treatment of compulsive eating disorders and obesity. Its action in overweight volunteers is associated with reduced consumption of, and reduced pleasurable response to, high fat/sugar snack items (Nathan et al, 2012). A functional magnetic resonance imaging study in healthy volunteers showed that, compared with NTX, GSK1521498 attenuated the activation in response to foodrelated stimuli of the amygdala and ventral striatum (Rabiner et al, 2011), brain areas that can be related to incentive salience and reward-reinforced behavior (Fletcher et al, 2010).

Here, we show that GSK1521498 effectively reduced both food-seeking and binge-like eating behaviors. These are two closely associated aspects of eating disorders, because food craving evoked by salient environmental stimuli can lead to loss of control over eating and potentially to overeating and obesity disorders. Obese individuals seem to be hyperresponsive to stimuli related to palatable food (Stice et al, 2008; Wang et al, 2002). As overeating disorders are multifaceted phenomena, GSK1521498 may provide a potential pharmacological treatment for binge eating disorders as it reduces both the propensity to seek preferred food maintained by CSs, as well as the tendency to eat sweet foods.

\section{ACKNOWLEDGEMENTS}

This work was supported by GSK. We thank Adam Mar for modifying the binge-eating med-associate procedure; Yann Pelloux for advice on conditioned place aversion experiments; Kristin Patterson and Ramprakash Govindarajan for providing the GSK1521498 solutions; Mark Bush for providing the data on the pharmacokinetic profile of GSK1521498; Sam Miller for critical review of the manuscript; and members of the GSK1521498 project team.

\section{DISCLOSURE}

CG was funded by GlaxoSmithKline (GSK). TWR has consulted for GSK, Cambridge Cognition, Lilly, Lundbeck, and Merck. He also has research grants with GSK, Lilly, and Lundbeck. ETB and PJN are employees of GSK and hold shares in the company. BJE has a research grant with GSK.

\section{REFERENCES}

Asarian L, Geary N (2006). Modulation of appetite by gonadal steroid hormones. Philos Trans $R$ Soc Lond B Biol Sci 361: 1251-1263.

Balleine B, Dickinson A (1992). Signalling and incentive processes in instrumental reinforcer devaluation. Q J Exp Psychol B 45: 285-301.

Barbano MF, Cador M (2006). Differential regulation of the consummatory, motivational and anticipatory aspects of feeding behavior by dopaminergic and opioidergic drugs. Neuropsychopharmacology 31: 1371-1381.
Bertino M, Beauchamp GK, Engelman K (1991). Naltrexone, an opioid blocker, alters taste perception and nutrient intake in humans. Am J Physiol 261: R59-R63.

Bodnar RJ (2004). Endogenous opioids and feeding behavior: a 30-year historical perspective. Peptides 25: 697-725.

Boggiano MM, Chandler PC (2006). Binge eating in rats produced by combining dieting with stress. Curr Protoc Neurosci, Chapter 9: Unit 9.23A.

Cleary J, Weldon DT, O'Hare E, Billington C, Levine AS (1996). Naloxone effects on sucrose-motivated behavior. Psychopharmacology 126: 110-114.

Corwin RL (2006). Bingeing rats: a model of intermittent excessive behavior? Appetite 46: 11-15.

Cottone P, Sabino V, Steardo L, Zorrilla EP (2008). Opioiddependent anticipatory negative contrast and binge-like eating in rats with limited access to highly preferred food. Neuropsychopharmacology 33: 524-535.

Drewnowski A, Krahn DD, Demitrack MA, Nairn K, Gosnell BA (1992). Taste responses and preferences for sweet high-fat foods: evidence for opioid involvement. Physiol Behav 51: 371-379.

Echo JA, Lamonte N, Ackerman TF, Bodnar RJ (2002). Alterations in food intake elicited by GABA and opioid agonists and antagonists administered into the ventral tegmental area region of rats. Physiol Behav 76: 107-116.

Everitt BJ, Fray P, Kostarczyk E, Taylor S, Stacey P (1987). Studies of instrumental behavior with sexual reinforcement in male rats (Rattus norvegicus): I. Control by brief visual stimuli paired with a receptive female. J Comp Psychol 101: 395-406.

Fantino M, Hosotte J, Apfelbaum M (1986). An opioid antagonist, naltrexone, reduces preference for sucrose in humans. Am J Physiol 251: R91-R96.

Flaherty CF, Coppotelli C, Grigson PS, Mitchell C, Flaherty JE (1995). Investigation of the devaluation interpretation of anticipatory negative contrast. J Exp Psychol Anim Behav Process 21: 229-247.

Fletcher PC, Napolitano A, Skeggs A, Miller SR, Delafont B, Cambridge VC et al (2010). Distinct modulatory effects of satiety and sibutramine on brain responses to food images in humans: a double dissociation across hypothalamus, amygdala, and ventral striatum. J Neurosci 30: 14346-14355.

Gandhi M, Aweeka F, Greenblatt RM, Blaschke TF (2004). Sex differences in pharmacokinetics and pharmacodynamics. Annu Rev Pharmacol Toxicol 44: 499-523.

Garbutt JC, Kranzler HR, O'Malley SS, Gastfriend DR, Pettinati HM, Silverman BL et al (2005). Efficacy and tolerability of longacting injectable naltrexone for alcohol dependence: a randomized controlled trial. JAMA 293: 1617-1625.

Glass MJ, Grace M, Cleary JP, Billington CJ, Levine AS (1996). Potency of naloxone's anorectic effect in rats is dependent on diet preference. Am J Physiol 271: R217-R221.

Ignar DM, Goetz AS, Noble KN, Carballo LH, Stroup AE, Fisher JC et al (2011). Regulation of ingestive behaviors in the rat by GSK1521498, a novel micro-opioid receptor-selective inverse agonist. J Pharmacol Exp Ther 339: 24-34.

Ito R, Robbins TW, McNaughton BL, Everitt BJ (2006). Selective excitotoxic lesions of the hippocampus and basolateral amygdala have dissociable effects on appetitive cue and place conditioning based on path integration in a novel Y-maze procedure. Eur J Neurosci 23: 3071-3080.

Johnson SW, North RA (1992). Opioids excite dopamine neurons by hyperpolarization of local interneurons. J Neurosci 12: 483-488.

Kelley AE (2004). Ventral striatal control of appetitive motivation: role in ingestive behavior and reward-related learning. Neurosci Biobehav Rev 27: 765-776.

Lenard NR, Zheng H, Berthoud H-R (2010). Chronic suppression of $[\mathrm{mu}]$-opioid receptor signaling in the nucleus accumbens 
attenuates development of diet-induced obesity in rats. Int $J$ Obes 34: 1001-1010.

Lynch WC (1986). Opiate blockade inhibits saccharin intake and blocks normal preference acquisition. Pharmacol Biochem Behav 24: 833-836.

Merrer J Le, Becker JAJ, Befort K, Kieffer BL (2009). Reward processing by the opioid system in the brain. Physiol Rev 89: 1379-1412.

Nathan PJ, Bullmore ET (2009). From taste hedonics to motivational drive: central $\mu$-opioid receptors and binge-eating behaviour. Int J Neuropsychopharmacol 12: 995-1008.

Nathan PJ, O’Neill BV, Bush MA, Koch A, Tao WX, Maltby K et al (2012). Opioid receptor modulation of hedonic taste preference and food intake: a single-dose safety, pharmacokinetic, and pharmacodynamic investigation with GSK1521498, a novel \{micro\}-opioid receptor inverse agonist. J Clin Pharmacol 52: 464-474.

Olszewski PK, Levine AS (2007). Central opioids and consumption of sweet tastants: when reward outweighs homeostasis. Physiol Behav 91: 506-512.

O’Malley SS, Krishnan-Sarin S, Farren C, O'Connor PG (2000). Naltrexone-induced nausea in patients treated for alcohol dependence: clinical predictors and evidence for opioidmediated effects. J Clin Psychopharmacol 20: 69-76.

Papaleo F, Kieffer BL, Tabarin A, Contarino A (2007). Decreased motivation to eat in mu-opioid receptor-deficient mice. Eur $J$ Neurosci 25: 3398-3405.

Peciña S, Berridge KC (2005). Hedonic hot spot in nucleus accumbens shell: where do mu-opioids cause increased hedonic impact of sweetness? J Neurosci 25: 11777-11786.

Pellegrini S, Wood M, Daniel AM, Papini MR (2005). Opioid receptors modulate recovery from consummatory successive negative contrast. Behav Brain Res 164: 239-249.

Petrovich GD, Ross CA, Gallagher M, Holland PC (2007). Learned contextual cue potentiates eating in rats. Physiol Behav 90: 362-367.

Pettinati HM, Kampman KM, Lynch KG, Suh JJ, Dackis CA, Oslin DW et al (2008). Gender differences with high-dose naltrexone in patients with co-occurring cocaine and alcohol dependence. J Subst Abuse Treat 34: 378-390.

Rabiner EA, Beaver J, Makwana A, Searle G, Long C, Nathan PJ et al (2011). Pharmacological differentiation of opioid receptor antagonists by molecular and functional imaging of target occupancy and food reward-related brain activation in humans. Mol Psychiatry 16: 826-835, 785.

Rozin P, Levine E, Stoess C (1991). Chocolate craving and liking. Appetite 17: 199-212.

Schultz W (2002). Getting formal with dopamine and reward. Neuron 36: 241-263.

Schultz W, Dayan P, Montague PR (1997). A neural substrate of prediction and reward. Science 275: 1593-1599.

Shin AC, Pistell PJ, Phifer CB, Berthoud HR (2010). Reversible suppression of food reward behavior by chronic mu-opioid receptor antagonism in the nucleus accumbens. Neuroscience 170: $580-588$.

Smith KS, Berridge KC (2005). The ventral pallidum and hedonic reward: neurochemical maps of sucrose 'liking' and food intake. J Neurosci 25: 8637-8649.

Smith KS, Berridge KC (2007). Opioid limbic circuit for reward: interaction between hedonic hotspots of nucleus accumbens and ventral pallidum. J Neurosci 27: 1594-1605.

Stice E, Spoor S, Bohon C, Veldhuizen MG, Small DM (2008). Relation of reward from food intake and anticipated food intake to obesity: a functional magnetic resonance imaging study. J Abnorm Psychol 117: 924-935.

Stoops WW (2008). Reinforcing effects of stimulants in humans: sensitivity of progressive-ratio schedules. Exp Clin Psychopharmacol 16: 503-512.

Wang G-J, Volkow ND, Fowler JS (2002). The role of dopamine in motivation for food in humans: implications for obesity. Expert Opin Ther Targets 6: 601-609.

Wansink B, Cheney MM, Chan N (2003). Exploring comfort food preferences across age and gender. Physiol Behav 79: 739-747.

Wassum KM, Ostlund SB, Maidment NT, Balleine BW (2009). Distinct opioid circuits determine the palatability and the desirability of rewarding events. Proc Natl Acad Sci USA 106: 12512-12517.

Weingarten HP (1983). Conditioned cues elicit feeding in sated rats: a role for learning in meal initiation. Science 220: 431-433.

Will MJ, Franzblau EB, Kelley AE (2003). Nucleus accumbens muopioids regulate intake of a high-fat diet via activation of a distributed brain network. J Neurosci 23: 2882-2888.

Williams KL, Broadbridge CL (2009). Potency of naltrexone to reduce ethanol self-administration in rats is greater for subcutaneous vs intraperitoneal injection. Alcohol 43: 119-126.

Woolley JD, Lee BS, Fields HL (2006). Nucleus accumbens opioids regulate flavor-based preferences in food consumption. Neuroscience 143: 309-317.

Yeomans MR, Gray RW (1997). Effects of naltrexone on food intake and changes in subjective appetite during eating: evidence for opioid involvement in the appetizer effect. Physiol Behav 62: $15-21$.

Yu Z, Geary N, Corwin RL (2008). Ovarian hormones inhibit fat intake under binge-type conditions in ovariectomized rats. Physiol Behav 95: 501-507.

Yu Z, Geary N, Corwin RL (2011). Individual effects of estradiol and progesterone on food intake and body weight in ovariectomized binge rats. Physiol Behav 104: 687-693.

Yun IA, Wakabayashi KT, Fields HL, Nicola SM (2004). The ventral tegmental area is required for the behavioral and nucleus accumbens neuronal firing responses to incentive cues. $J$ Neurosci 24: 2923-2933.

Zhang M, Balmadrid C, Kelley AE (2003). Nucleus accumbens opioid, GABaergic, and dopaminergic modulation of palatable food motivation: contrasting effects revealed by a progressive ratio study in the rat. Behav Neurosci 117: 202-211.

Zhang M, Gosnell BA, Kelley AE (1998). Intake of high-fat food is selectively enhanced by mu opioid receptor stimulation within the nucleus accumbens. J Pharmacol Exp Ther 285: 908-914.
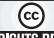

OMERIGHIS RESERVED

This work is licensed under the Creative Commons Attribution-NonCommercial-No Derivative Works 3.0 Unported License. To view a copy of this license, visit http://creativecommons.org/ licenses/by-nc-nd/3.0/

Supplementary Information accompanies the paper on the Neuropsychopharmacology website (http://www.nature.com/npp) 